\title{
Fatores de risco e de proteção para o uso de drogas na adolescência
}

\author{
Risk and protective factors and drug \\ use among adolescence
}

M iriam Schenker 1

M aria Cecília de Souza Minayo 2

\footnotetext{
1 Núcleo de Estudos e Pesquisa em Atenção ao U so de Drogas/UERJ, Pós-Graduação em Saúde da Criança e da M ulher do Instituto Fernandes Figueira, Fiocruz. Rua Fonseca Teles 121/40 andar, 20940-200, Rio de Janeiro RJ. schenkerbrasil@hotmail.com 2 Centro Latino-

Abstract This article presents a comprehensive view of the risk and protective factors complexity to drug use in adolescence. Discusses the interdependence of various contexts - individual, familiar, school, peer group, midia and community favourable either to risk or protection of licit and illicit drug use, providing, finally, some prevention strategies.

Key words Adolescence, Risk factors, Protective factors, Drug use, Prevention
Resumo Este artigo apresenta uma visão compreensi va da complexidade dos fatores de risco e de proteção para o uso de drogas na adolescência. Discorre sobre a interdependência dos diversos contextos - individual, familiar, escolar, grupo de pares, midiático e comunidade de convivência propícios tanto ao risco quanto à proteção ao uso das drogas lícitas e ilícitas, fornecendo, por último, algumas estratégias de prevenção.

Palavras-chave Adolescência, Fatores de risco, Fatores de proteção, U so de drogas, Prevenção 


\section{Introdução}

N este artigo discute-se a prevenção ao uso indevido de drogas por adolescentes. Analisam-se os fatores de risco e os fatores de proteção, conceitos que servem de base para o diálogo com os diferentes contextos sociais como a família, os pares, a escola, a comunidade e a mídia.

A adolescência constitui um período crucial no ciclo vital para o início do uso de drogas, seja como mera experimentação seja como consumo ocasional, indevido ou abusivo. Esse estudo privilegia a discussão da prevenção, lembrando que a utilização das drogas lícitas e ilícitas permeia a cultura da adolescência à velhice e, no caso do Brasil, notadamente por meio do consumo de álcool, fumo e maconha.

A família, pelo papel de inserir seus membros na cultura e ser instituidora das relações primárias, influencia a forma como 0 adolescente reage à ampla oferta de droga na sociedade atual. Relações familiares saudáveis desde o nascimento da criança servem como fator de proteção para toda a vida e, de forma muito particular, para o adolescente. No entanto, problemas enfrentados na adolescência, plantados na infância, têm um contexto de realização muito mais ampliado. Buscar-se-á, portanto, neste texto, realizar uma discussão do contexto familiar e outros ambientes importantes para a prevenção do uso indevido de drogas, como é o caso do grupo de pares, da escola, da comunidade e da mídia.

\section{Material e método}

A elaboração deste artigo foi precedida de pesquisas nas bases de dados PubM ed, M edline e de outros textos requisitados diretamente aos autores, entre os anos de 1995 e 2003, a partir das seguintes palavras-chave: adolescência (adolescence), fatores de risco (risk factors), fatores de proteção (protective factors), uso de drogas (drug use), prevenção (prevention). Outras obras e artigos são citados apenas quando relevantes ao tema em questão. Os 67 textos de referência foram analisados, comparados e avaliados na íntegra, dentro do escopo do presente estudo.

0 ponto de vista metodológico aqui adotado é o da teoria sistêmica/ecológica, coincidente com o de praticamente todos os autores de referência. 0 foco da análise se detém nas relações intrafamiliares e interpessoais, contextualizadas socioculturalmente.

\section{Discussão teórica}

Este artigo se elabora em torno de dois conceitos centrais: risco e proteção. E ao redor deles, trabalham-se as noções de adolescência, família, escola, grupo de pares, comunidade e mídia como espaços fundamentais para a construção de risco e de proteção.

\section{Risco e fatores de risco}

Risco é uma conseqüência da livre e consciente decisão de se expor a uma situação na qual se busca a realização de um bem ou de um desejo, em cujo percurso se inclui a possibilidade de perda ou ferimento físico, material ou psicológico. De acordo com Pieper \& Pieper (1999) o ser humano que possui a virtude cardeal da fortaleza expõe-se ao perigo da morte por um bem (1999). Segundo M cCrimmond \& Werhrung (1986), existem três condições para a definição de risco: (1) possibilidade de haver perda; (2) possibilidade de ganho; e (3) possibilidade de aumentar ou de diminuir a perda ou os danos. Segundo grandes filósofos como H eidegger, o risco é inerente à vida (1980), ao movimento, eà possibilidade de escolha. Viver é correr risco e por isso a incerteza é um componente essencial da existência e igualmente do conceito de risco.

Giddens (1994) faz uma distinção interessante entre risco e perigo. Comenta 0 autor que esses dois termos não são sinônimos embora seu significado se aproxime. Perigo diz respeito a ameaças que rondam a busca dos resultados desejados. Risco constitui uma estimativa acerca do perigo.

$\mathrm{Na}$ área de saúde, risco é um conceito que envolve conhecimento e experiência acumulada sobre o perigo de alguém ou de a coletividade ser acometida por doenças e agravos. Sendo um termo central da epidemiologia, diz respeito a situações reais ou potenciais que produzem efeitos adversos e configuram algum tipo de exposição. Definidos a partir de análises coletivas, os alertas trazidos à população pela epidemiologia se aplicam a cuidados e evitação.

A expressão consagrada fatores de risco de signa condições ou variáveis associadas à possibilidade de ocorrência de resultados negativos para a saúde, o bem-estar e o desempenho social (N ewcomb et al., 1986; Jessor, 1991; Jessor et al., 1995). Alguns desses fatores se referem a características dos indivíduos; outros, ao seu meio microssocial e outros, ainda, a condições estru- 
turais e socioculturais mais amplas (Zweig et al., 2002), mas, geralmente, estão combinados quando uma situação considerada social, intrapsíquica e biologicamente perigosa se concretiza. Por exemplo, no caso do uso de drogas: ao fumar maconha, 0 adolescente pode aumentar a probabilidade de desenvolver uma doença pulmonar, e também sofrer conseqüências psicossociais ou sanções legais, conflitos com os pais, perda de interesse na escola ou culpa e ansiedade.

Sobretudo quando se trabalha com adolescentes, o conceito de risco tal como visto pela epidemiologia não é suficiente, uma vez que nessa ótica é entendido, apenas, segundo suas conseqüências negativas. No exemplo dado acima, está claro que um adolescente que usa maconha em princípio busca prazer e não dor e sofrimento. Em geral está a cata de extroversão, prazer, novas sensações, compartilhamento grupal, diferenciação, autonomia e independência em relação à família, dentre outros efeitos. $\mathrm{E}$ nessa procura faz um cálculo do perigo a que se expõe. Os profissionais que atuam na prevenção precisam saber desse outro lado da questão, sob pena de não desenvolverem uma compreensão suficientemente ampla e profunda do fenômeno do uso de drogas.

0 lado negativo do desejo juvenil de obter prazer com o uso de drogas é o risco que ele corre de se tornar dependente e comprometer a realização de tarefas normais do desenvolvimento; o cumprimento dos papéis sociais esperados; a aquisição de habilidades essenciais; a realização de um sentido de adequação e competência e a preparação apropriada para a transição ao próximo estágio na trajetória da vida: 0 adulto jovem. 0 termo comportamento de risco aqui, portanto, se refere às ameaças ao desenvolvimento bem-sucedido do adolescente. U ma preocupação básica da educação para a saúde seria, pois, discutir com os adolescentes os riscos associados aos comportamentos nos quais se engajam (J essor, 1991), mas tendo o cuidado de não desconhecer o lado prazeroso desse engajamento.

A necessidade de se olhar os dois lados, o do desejo e o do dano, no caso do uso de drogas, leva a considerar al guns aspectos citados a seguir.

(1) Um deles diz respeito aos efeitos cumulativos das substâncias tóxicas e sua relação com a vulnerabilidade do indivíduo. De um lado sabe-se que a probabilidade de desenvolvimento de determinado distúrbio aumenta em função do número, da duração e da "toxicidade" dos fatores de risco envolvidos (Coie et al.,
1993). Por isso, quanto mais intenso o uso de drogas, mais fatores de risco há (Bry et al., 1982; N ewcomb et al., 1986). Por outro lado, vários estudos mostram que o perigo difere de acordo com os indivíduos e seu contexto. Kandel e colaboradores (1978) enfatizam que os adolescentes em certo estágio e freqüência de consumo não necessariamente irão usar drogas mais pesadas. Essa constatação vai contra a idéia de que haveria uma certa seqüência na gravidade do risco, indo do envolvimento sucessivo com substâncias mais leves para uma escalada rumo às mais nocivas. Alguns estudos chegam a sugerir que cigarro e álcool funcionariam como ponte (gateway) para um caminho progressivo de envolvimento com drogas cada vez mais pesadas (De M icheli \& Formigoni, 2002). Essa teoria tem conseqüência para a prática educativa que busca dissuadir os adolescentes de usar substâncias mais leves, visando a prevenir o uso de outras (Botvin, 1986). No entanto, na atualidade, mesmo os estudiosos que continuam a afirmar o risco de exposição crescente, dizem que a relação é de probabilidade e não causal (Ellickson \& M orton, 1999).

(2) Um segundo aspecto importante a ser considerado éo que se refere ao risco que constitui a atitude positiva da família com relação ao uso de drogas, reforçando a iniciação dos jovens (Kandel et al., 1978). H oje se sabe que as relações familiares constituem um dos fatores mais relevantes a ser considerado, mas de forma combinada com outros. Por exemplo, Schor (1996) aponta que não há uma relação linear entre 0 abuso de álcool dos pais e de seus fiIhos. Sugere que os padrões de comportamento dos pais e as interações familiares, e não só 0 fato de eles beberem, são em boa parte responsáveis pelas atitudes dos filhos. 0 alcoolismo tem uma influência destrutiva no funcionamento familiar e essa disfunção desempenha um papel mediador na transmissão intergeracional de comportamentos. Mas, o que está em questão não é a droga em si, e sim, a relação que o indivíduo estabelece com ela, que, por sua vez, influencia e é influenciada fortemente pelo universo de interações. Embora o consumo de drogas pelos pais esteja relacionado a maior risco de os filhos se tornarem usuários, uma vez que o comportamento parental lhes serve de modelo, é a atitude permissiva dos genitores o que mais pesa nessa equação ( $\mathrm{H}$ awkins et al., 1992; Brown et al., 1993).

Estudos têm mostrado que os fatores parentais de risco para o uso de drogas pelo ado- 
lescente incluem, de forma combinada: (a) ausência de investimento nos vínculos que unem unem pais e filhos ( $H$ awkins et al., 1992; Patton, 1995; Kodjo \& Klein, 2002); (b) envolvimento materno insuficiente (Tarter et al., 2002); (c) práticas disciplinares inconsistentes ou coercitivas (Friedman, 1989; Brook et al., 1990; H awkins et al., 1992; Patton, 1995); (d) excessiva permissividade, dificuldades de estabelecer limites aos comportamentos infantis e juvenis e tendência à superproteção; (e) educação autoritária associada a pouco zelo e pouca afetividade nas relações (Tuttle et al., 2002; Patton, 1995); (f) monitoramento parental deficiente (H awkins et al., 1992); (g) aprovação do uso de drogas pelos pais (Friedman, 1989; H awkins et al., 1992); (h) expectativas incertas com relação à idade apropriada do comportamento infantil (Tarter et al., 2002); (i) conflitos familiares sem desfecho de negociação ( $H$ awkins et al., 1992; Patton, 1995; Kodjo \& Klein, 2002).

(3) $O$ envolvimento grupal tem sido visto como um dos maiores prenúncios do uso de substâncias (Kandel et al., 1978; Botvin, 1986; Brook et al., 1990; H awkins et al., 1992; O etting \& Donnermeyer, 1998; Fergusson \& H orwood, 1999; Swadi, 1999; H offmann \& Cerbone, 2002). No entanto, essa relação interpares também precisa ser qualificada. Ela se configura como fator de risco quando os amigos considerados modelo de comportamento (Jessor et al., 1995; H offmann \& Cerbone, 2002; Swadi, 1999) demonstram tolerância, aprovação (Kodjo \& Klein, 2002) ou consomem drogas (Beman, 1995). O bservam os estudiosos que há uma sintonia, no caso dos pares: os adolescentes que querem começar ou aumentar o uso de drogas procuram colegas com valores e hábitos semelhantes (Tuttle et al., 2002). Ou seja, mesmo no caso de amigos e colegas, a questão não pode ser vista de forma simplista, pois o desenvolvimento de afiliações a pares tolerantes e que aprovam as drogas representa o final de um processo onde fatores individuais, familiares e sociais adversos se combinam de forma a aumentar a probabilidade do uso abusivo (Fergusson \& $\mathrm{Ho}$ wood, 1999). 0 mito que supervaloriza a influência dos pares durante a adolescência provavelmente decorre, em algum nível, de uma certa desresponsabilização, sobretudo por parte dos pais e dos educadores, de problemas freqüentes nas relações intrafamiliares ou institucionais. É muito difícil, portanto, como já se referiu, tomando por base outros fatores, separar e isolar os efeitos que o grupo de pares tem so- bre os adolescentes, embora se saiba que seu poder é importante no caso do uso de drogas (Schor, 1996).

(4) M uito se tem falado também no papel da escola seja como agente transformador, seja como lócus propiciador do ambiente que exacerba as condições para o uso de drogas. Ninguém desconhece que essa instituição é hoje alvo do assédio de traficantes e repassadores de substâncias proibidas, prevendo-se 0 aliciamento por pares. Pois a escola é o espaço privilegiado dos encontros einterações entre jovens. No entanto, mesmo no âmbito educacional, existem fatores específicos que predispõem os adolescentes ao uso de drogas, como por exemplo, a falta de motivação para os estudos, 0 absenteísmo e o mau desempenho escolar (Kandel et al., 1978); a insuficiência no aproveitamento e a falta de compromisso com o sentido da educação; a intensa vontade de ser independente, combinada com o pouco interesse de investir na realização pessoal (Friedman, 1989); a busca de novidade a qual quer preço e a baixa oposição a situações perigosas; a rebeldia constante associada à depen dência a recompensas (Swadi, 1999).

(5) A disponibilidade e a presença de drogas na comunidade de convivência têm sido vistas como facilitadoras do uso de drogas por adolescentes, uma vez que o excesso de oferta naturaliza o acesso (J essor, 1991; Patton, 1995; Wallace Jr., 1999). Quando a facilidade da oferta se junta à desorganização social e aos outros elementos predisponentes no âmbito familiar e institucional, produz-se uma sintonia de fatores (Kodjo \& Klein, 2002). A observação sobre esse elemento, privilegiando-o e ao mesmo tempo associando-o aos outros é importante pois permite correlacionar fatos como gravidez precoce e evasão escolar (H awkins et al., 1992), acidentes de carro, homicídios e suicídios (Kodjo \& Klein, 2002).

(6) Outra tendência muito comum quando se fala de drogas é a absolutização do papel da mídia como fator de risco. É certo que, sobretudo no caso das drogas lícitas, os meios de comunicação geralmente mostram imagens muito favoráveis. 0 uso do álcool e do tabaco costuma vir associado, por meio da publicidade, a imagens de artistas, ao glamour da sociabilidade e à sexualidade. Freqüentemente os anúncios glorificam as substâncias, retratando-as como mediadoras de fama e sucesso (Patton, 1995; Kodjo \& Klein, 2002; M inayo et al., 1999; Njaine, 2004). M as não se pode, teoricamente, 
demonizar a mídia: de um lado ela reflete e refrata a cultura vigente. $E$, de outro, seria um erro menosprezar a capacidade crítica dos jovens e a sinergia de vários outros elementos com os meios de comunicação. Nenhuma propaganda por si só atinge efeito demoníaco de persuasão, quando fatores protetores atuam em direção contrária. 0 desenvolvimento de um espírito crítico er reflexivo na família, na escola e com os pares serve de base para uma atitude criteriosa do adolescente quanto às mensagens relativas às drogas lícitas, veiculadas pelos meios de comunicação.

Os diversos elementos tratados acima levam a concluir que não se pode pensar os fatores de risco de forma isolada, independente e fragmentada. Determinado fator de risco raramente é específico de um distúrbio único, porque seus contextos formadores ten dem a espaIhar os efeitos dele derivados sobre uma série de funções adaptadoras ao longo do desenvolvimento. E a exposição ao perigo que potencializa os riscos ocorre de diversas formas e em vários contextos, como por exemplo: exacerbando fatores individuais, educação infantil insatisfatória, fracassos escolares, relações sociais problemáticas entre os pares ou com desorganização da comunidade ( $N$ ewcomb et al., 1986; Coie et al., 1993; H awkins et al., 1992; Patton, 1995). M esmo a mais elevada carga genética é menos provável de se constituir em alto fator de risco numa sociedade na qual a exposição ao ál cool seja severamente restrita, comenta Swadi (1999). A postura unicausal, tão freqüente, é responsável por práticas, orientações e criação de instituições inócuas ou nocivas, às quais falta uma lógica compreensiva e sistêmica.

Ressaltando a necessidade metodológica de levar em conta a complexidade das formas de adesão ao uso abusivo de drogas na adolescência é rel evante pensar que há uma estrutura e uma organização dos diferentes fatores, formando uma síndrome do comportamento de ris$\mathrm{Co}, 0$ que remete às premissas do paradigma sistêmico: os comportamentos de risco co-variam e estão inter-relacionados. N essa perspectiva, os esforços preventivos devem visar a modificar as circunstâncias que sustentam tais síndromes: o estilo de vida que sintetiza um padrão organizado de comportamentos inter-relacionados, dentro de uma ecologia social (Jessor, 1991) e em relações complexas com distúrbios clínicos.

\section{Proteção e fatores de proteção}

Proteger é uma noção que faz parte do contexto das relações primárias e do universo semântico das políticas sociais. Significa, sobretudo, oferecer condições de crescimento e de desenvolvimento, de amparo e defortalecimento da pessoa em formação. No caso brasileiro, a doutrina da proteção integral se encontra no Estatuto Brasileiro da Criança e do Adolescente (ECA), que a resume definindo esse grupo social como (a) cidadão; (b) sujeito de direitos; (c) capaz de protagonismo; (d) merecedor de prioridade de atenção; e (e) de cuidados.

Dentro dessa premissa de proteção, uma das tarefas de quem atua na atenção aos adolescentes que usam drogas é determinar que fatores podem ser evidenciados pela técnica epela experiência como relevantes para promover seu crescimento saudável e evitar que corram riscos de dependências e de acirramento de problemas sociais (H awkins et al., 1992; Coie et al., 1993; Jessor et al., 1995; Pettit et al., 1997). Cabe ressaltar que os fatores de risco e de proteção devem ser tratados como variáveis independentes, pois podem afetar o comportamento sem que haja, necessariamente, uma complementaridade entre eles (Jessor et al., 1995).

0 desenvolvimento dos estudos sobre fatores protetores, tende, atualmente, a enfatizar 0 processo de formação da resiliência, num progressivo abandono das abordagens centradas nos fatores de risco. Busca-se dar ênfase aos elementos positivos que levam um indivíduo a superar as adversidades. Esse novo paradigma é certamente otimista, principalmente porque leva a acreditar que é possível, por meio de ações e programas, promover o bem-estar do adolescente, atuando no fortalecimento e no desenvolvimento de habilidades pessoais e sociais (M unist et al., 1998; Bloom, 1996; Assis, 1999; Assis \& Constantino, 2001).

Embora as definições de resiliência sejam ainda bastante variadas, toda a discussão a respeito desse conceito está relacionada aos fatores ou processos intrapsíquicos e sociais que possibilitem o desenvolvimento de uma vida sadia, apesar de experiências de vida traumáticas. A compreensão do conceito envolve $o$ entendimento da interação entre a adversidade e fatores de proteção internos e externos ao sujeito, assim como do desenvolvimento de competências que permitam a uma pessoa obter sucesso diante da adversidade (Rutter, 1987; Assis, 1999). 
Diante de eventos traumáticos, os elementos de proteção assumem papel facilitador no caminho da construção da resiliência. Os estudiosos têm identificado três categorias de fatores de proteção em crianças e adolescentes resilientes: (a) individuais: temperamento que favoreça o enfrentamento do problema, autoimagem positiva e a capacidade de criar e desenvolver estratégias ativas na forma de lidar com problemas. Esses atributos denotam autoeficácia, autoconfiança, habilidades sociais e interpessoais, sentimentos de empatia, controle emocional, humor e relacionamento com os pares. Os estudos mostram que existem especificidades de gênero, de idade e de raça nas formas de demonstração da resiliência; (b) familiares: que se traduzem em suporte, segurança, bom relacionamento e harmonia com pais e no ambiente de relações primárias; (c) extrafamiliares ou ambientais, quando se referem ao suporte de pessoas significativas e experiências escolares positivas (Emery \& Forehand, 1996; Werner, 1996; Assis, 1999; Assis \& Constantino, 2001).

A forma como o indivíduo lida com as adversidades, em psicologia, é chamada coping, termo que agrega o conjunto de estratégias utilizadas pelas pessoas diante de circunstâncias adversas ou estressantes. 0 coping positivo é construído ao longo do tempo edo processo de crescimento e desenvolvimento individual. Tal como a resiliência, as estratégias de coping dependem de atributos individuais, familiares e ambientais para se consolidarem no indivíduo. U ma vez estabelecidas, as estratégias funcionam como importante fator de proteção ao risco, proporcionando resiliência, caso sejam predominantemente ativas no sentido da resolução dos problemas (Garmezy \& Rutter, 1988). Ao contrário, quando o indivíduo vai consolidando estratégias de fuga e evitação dos problemas, elas se tornam fatores prejudiciais ao seu desenvolvimento saudável. Ambos dizem respeito a como os fatores familiares, micro e macrossociais e estruturais atingem 0 indivíduo e o tornam em mais suscetível às influências adversas, ou mais resistente a seus influxos. No caso do uso de drogas, essa perspectiva de resiliência deve ser adotada para buscar formas de reduzir a vulnerabilidade do indivíduo (H awkins et al., 1992; Swadi, 1999).

0 estudo de Brook e colaboradores (1990) aborda dois mecanismos através dos quais os fatores de proteção reduziram o risco do uso de drogas pelo adolescente. 0 primeiro é um me- canismo risco/proteção por meio do qual a exposição aos fatores de risco é moderada pela presença de fatores de proteção. Em estudo empírico, os autores mostram que o risco colocado por amigos que usavam drogas foi moderado pela forte inter-relação entre os pais e os adolescentes e pelo comportamento convencional e formal dos pais. 0 outro é um mecanismo proteção/proteção segundo o qual um fator potencializa outro, tornando o seu efeito mais forte. Por exemplo, os autores observaram que o vínculo entre 0 adolescente e seu pai intensificou os efeitos protetores, tais como o comportamento formal do adolescente, as características maternas positivas e a harmonia marital, atuando positivamente na prevenção ao uso de droga.

Fatores de proteção são identificados nos seis domínios da vida já tratados, quando se discutiu a questão dos riscos: individual - atitudes e predisposições; meio familiar - relações familiares e atitudes parentais; escola - clima seguro ou inseguro; amigos - envolvimento ou não com drogas; sociedade - tendências econômicas, falta de emprego; comunidade- organização ou desorganização (Zweig et al., 2002).

Olhando os aspectos da individualidade, é preciso ressaltar que os adolescentes não são um recipiente passivo ou objeto controlado por influências familiares ou sociais e nem por determinações externas. São participantes ativos do processo de formação de vínculos e de transmissão de normas. Suas características físicas, emocionais e sociais interagem na dinâmica de socialização (O etting et al., 1998), permitindo a metabolização subjetiva dos fatores externos. Por exemplo: em geral as crianças com nove ou dez anos valorizam a companhia dos amigos, as atividades de grupo, o sucesso na escola, a boa relação com os pais. Porém, cada menino ou menina avalia de forma diferente a rejeição dos amigos ou sua exclusão de um grupo. Para uma criança, a experiência da rejeição pode ter efeitos deletérios imediatos na cognição social, na auto-imagem e na auto-estima. Para outra, a mesma experiência pode ter efeitos saudáveis no ajustamento de longo prazo, motivando-a a se posicionar de forma a ser aceita no futuro (Parker $\&$ Asher, 1987; Tarter et al., 2002).

Os adolescentes que têm objetivos definidos e investem no futuro apresentam probabilidade menor de usar drogas, porque o uso interfere com os seus planos (Kodjo \& Klein, 2002). I gualmente, a elevada auto-estima, os 
sentimentos de valor, orgulho, habilidade, respeito e satisfação com a vida podem servir de proteção aos jovens contra a dependência de drogas quando combinada com outros fatores protetores do seu contexto de vida (H offmann $\&$ Cerbone, 2002). Sendo assim, conclui-se que crianças e adolescentes que vivem em ambientes familiares ou em comunidades onde há uso abusivo de drogas e conseguem não se deixar influenciar por esse contexto apresentam características individuais protetoras conjugadas ao convívio com outros adultos cuidadores escolhidos por eles, fora do ambiente familiar. Os programas de prevenção devem levar em conta a importância das atividades de mentores e de outros programas de desenvolvimento da juventude (Kodjo \& Klein, 2002).

0 âmbito familiar tem um efeito potencialmente forte e durável para o ajustamento infantil. 0 vínculo e a interação familiar saudável servem de base para o desenvolvimento pleno das potencialidades das crianças e dos adolescentes. I números estudos mostram que os padrões de relação familiar, a atitude e o comportamento dos pais e irmãos são modelos importantes para os adolescentes, inclusive no caso do uso de drogas (Kandel et al., 1978; Brook et al., 1990; H awkins et al., 1992; Patton, 1995; Schor, 1996; Kodjo \& Klein, 2002). Tec (1974) descobriu que uma interação familiar gratificante é um forte fator protetor, mesmo no caso dos pais adictos, quando esses são capazes de prover um contexto amoroso, afetuoso e de cuidado.

No âmbito da família, estudos evidenciam como fatores que protegem o adolescente do uso de drogas: (a) a relevância dos vínculos familiares fortes (Kandel et al., 1978; Swadi, 1999; Werner et al., 1999; Hoffmann \& Cerbone, 2002; Schenker \& M inayo, 2003); (b) o apoio da família ao processo de aquisição da autonomia pelo adolescente (Tuttle et al., 2002); (c) o monitoramento parental aos diversos processos de crescimento e desenvolvimento (Steinberg et al., 1994; Chilcoat \& Anthony, 1996; Swadi, 1999; Patton, 1995; Werner et al., 1999; Brook et al., 1990); (d) o estabelecimento de normas claras para os comportamentos sociais, incluindose 0 uso de drogas ( 0 etting $\&$ Donnermeyer, 1998).

Raramente os estudos sobre drogas dão realce às amizades entre os jovens como protetoras, uma vez que, em geral, todas as intervenções focalizam a superação das influências negativas das amizades e não o estabelecimento ou manutenção de influências positivas dos amigos (Tuttle et al., 2002). No entanto, sabese que grupos de amigos com objetivos e expectativas de realização na vida e movimentos que levam ao protagonismo juvenil e à solidariedade têm papel fundamental numa etapa existencial em que as influências dos pares são cruciais.

A escola é um poderoso agente de socialização da criança e do adolescente, ressaltandose uma certa mística e identidade do tipo de educandário com o comportamento daqueles que o freqüentam (Kandel et al., 1978). Por juntar em seu interior a comunidade de pares e por ter fortes instrumentos de promoção da auto-estima e do autodesenvolvimento em suas mãos, 0 ambiente escolar pode ser um fator fundamental na potencialização de resiliência dos adolescentes.

No que concerne aos fatores estressantes da vida, como morte, doenças ou acidentes entre membros da família e amigos; mudanças de escola ou de residência; separação, divórcio ou novos casamentos dos pais; problemas financeiros na família (H offmann \& Cerbone, 2002), muitos estudos mostram que eles podem influenciar o uso abusivo de drogas quando associados a outros fatores predisponentes, incluindo-se disposições individuais. No entanto, conforme as circunstâncias individuais e ambientais, eles permitem elaboração e crescimento interior dos jovens, constituindo-se em elementos de fortal ecimento e de amadurecimento.

Os adolescentes são consumidores ávidos da mídia escrita e audiovisual. As mensagens recebidas desses meios geralmente influenciam sua tomada de decisão a respeito de vários assuntos em sua vida. Entretanto, a reflexão crítica deles entre pares e com pais e educadores moderam o risco potencial da exposição e potencializam a comunicação e o amadurecimento em relação aos vários problemas, inclusive sobre o uso de substâncias ilícitas.

Por tudo o que foi dito até então, é preciso refletir sobre a inocuidade, do ponto de vista protetor, do slogan que se repete por toda parte "diga não às drogas". A falta de adesão dos jovens torna evidente a fal ha em reconhecer a inadequação das propostas moralistas e autoritárias que não se fundam na visão complexa dos fatores de risco e de proteção analisados. É preciso não se esquecer que as drogas cumprem funções importantes para os adolescentes, tanto do ponto de vista pessoal quanto social. Pesquisas mostram que os comportamentos de en- 
fretamento de risco são funcionais, intencionais, instrumentais e dirigidos para o desenvolvimento normal do adolescente. Fumar, beber, dirigir perigosamente ou exercer atividade sexual precocemente podem ser atitudes tomadas pelo jovem visando a ser aceito e respeitado pelos pares; conseguir autonomia em relação aos pais; repudiar normas e valores da autoridade convencional; lidar com ansiedade, frustração e antecipação do fracasso; afirmação rumo à maturidade e à transição da infância para um status mais adulto. Não há nada de perverso, irracional ou psicopatológico nesses objetivos: eles são característicos do desenvolvimento psicossocial. A campanha "diga não às drogas", por não oferecer alternativas à promoção de comportamentos saudáveis, revela-se moralmente cínica (Shedler \& Block, 1990; Jessor, 1991) e teoricamente contraditória, na me dida em que omite as normas sociais que favorecem o uso de drogas (Beman, 1995).

\section{Estratégias de prevenção}

0 adolescente que faz uso de drogas responde bem às intervenções contextualizadas. O s contextos dominantes para ele são seus pares e a escola e, numa proporção menor, o entorno da comunidade. Para crianças menores, o contexto principal éa família, que continua igualmente importante na adolescência (Schor, 1996).

O uso ocasional de droga por adolescentes pode ser entendido como manifestação de uma experimentação apropriada para sua etapa de desenvolvimento e busca de direção para a vida. Estudo de Shedler e Block (1990), ao diferençar entre adolescentes que se abstêm, que experimentam e que abusam de drogas, conclui que, dada a quase onipresença e uma certa aceitação da maconha na cultura dos pares, não é surpreendente que, com 18 anos, indivíduos psicologicamente saudáveis, sociáveis e curiosos se sintam tentados a experimentar 0 produto. Não se espera que adolescentes saudáveis abusem da droga, porque apresentam baixa necessidade de utilizá-la para aplacar a angústia emocional ou como meio de compensar a falta de relações importantes. Os autores comparam o sentido do uso da maconha entre adolescentes de hoje com o significado social e psicológico que a bebida alcoólica teve para gerações anteriores (Shedler \& Block, 1990). Levando em conta essa reflexão, os esforços preventivos precisam ser muito mais abrangentes e voltados para os precursores dos riscos e da resiliência (Coie et al., 1993).

Estudos indicam que as condições de formação de uma personalidade resiliente são: (a) colocar expectativas claras relativas ao comportamento; (b) monitorar e supervisionar as crianças; (c) reforçar com consistência atividades que favoreçam a socialização; (d) criar oportunidades para o envolvimento familiar; (e) promover o desenvolvimento das habilidades acadêmicas e sociais dos jovens ( $H$ awkins et al., 1992).

No entanto, as habilidades para a formação de crianças e jovens freqüentemente deveriam fazer parte de um processo de formação de pais e educadores. A aquisição e o uso dessas habilidades na administração da família ou dos contextos educativos reduzem problemas de comportamento das crianças nos primeiros anos, promove o bom desempenho escolar e as fortalece para lidarem com condições adversas (H awkins et al., 1992).

Pela força da escola e da família nessa etapa da vida, para os pré-adolescentes e adolescentes é fundamental que pais e educadores estejam atentos a alguns parâmetros relacionais: (a) uma comunicação livre e fluente com os pais ou com adultos que lhes servem de modelo fortalece emocionalmente o jovem; (b) e evita 0 engajamento em comportamentos de risco (Friedman, 1989; Kodjo \& Klein, 2002; Tuttle, 2002); (c) elogios dos pais às conquistas dos fiIhos e dos educadores a seus estudantes são o alimento da auto-estima; (d) a colocação de expectativas claras por parte dos pais e professores, aliada a uma educação com autoridade, que envolve afeto, controle e trato democrático, favorece o desenvolvimento psicológico saudável e o sucesso escolar do adolescente (Steinberg et al., 1989; 1992); (e) o monitoramento das atividades dos jovens, seja por pais ou educadores, mostra que eles estão investindo na segurança dos jovens; (f) o compartilhamento de valores, atitudes e crenças sobre as drogas (caso em pauta aqui) é fundamental para o amadurecimento das decisões e da responsabilização; (g) conhecer os amigos e os pais dos amigos dos filhos é crucial, uma vez que a pressão dos pares é uma das principais influências para o uso de drogas; (h) exigências e expectativas quanto ao desempenho na escola funcionam como um tipo de monitoramento e de proteção, na medida em que se juntam ao encorajamento de atividades em que o jovem possa ter sucesso; (i) por fim, o incentivo ao engajamento 
nas atividades da escola, da comunidade e de movimentos sociais ou de solidariedade é um potente fator protetor.

Para Hawkins e colaboradores (1992), os quatro elementos do vínculo social quese mostram inversamente correlacionados com o uso de drogas são: (a) vínculo forte com os pais; (b) compromisso com a escola; (c) envolvimento regular com atividades da igreja ou de outros movimentos; (d) crença nas expectativas gerais, normas e valores da sociedade.

M etodologicamente, essas intervenções requerem intercomunicação com a criança, os pais, a família e a comunidade. Já que influência dos pais diminui à medida que as crianças crescem, o dito popular quanto mais cedo meIhor deve guiar os programas de intervenção. A ênfase no estilo de vida nunca deve ser traduzida como se esse estilo fosse de responsabilidade exclusiva do indivíduo: tal abordagem tenderia a culpar a vítima (Jessor, 1991). 0 risco precisa ser entendido, como já foi dito, de forma a incluir a complexidade dos fatores e, portanto, a redução do risco requer tanto mudanças subjetivas quanto sociais.

Pode-se esperar um maior sucesso dos programas de prevenção que explicitamente maximizam o ajuste adaptativo entre indivíduos e contextos. Para tal, é necessário ter sensibilidade para compreender e valorizar a história pessoal, o estágio de vida do indivíduo, as normas culturais, as crenças e práticas no que concerne ao uso de drogas (Tec, 1974; Johnson et al., 1984; Coie et al., 1993), bem como os contextos sociais e da comunidade onde esse uso ocorre ou é proscrito (Wallace Jr., 1999).

\section{Conclusão}

O uso de drogas é uma questão complexa que perpassa inúmeros subsistemas da vida individual e social. As representações sociais que levam à adesão ou à condenação dependem do contexto sociocultural. Os constrangimentos impostos numa determinada cultura são diversos em outras. Então, é necessário compreender os códigos do contexto e a rede de significados que envolvem a sociedade em geral, os grupos específicos dentro de determinado tempo histórico. M uitos estudos têm mostrado como a atribuição de valor positivo ou proscriti- vo dependem de configurações geográficas e históricas (Velho, 1998).

Este artigo inicialmente privilegiou o papel da família na prevenção e na promoção da resiliência, porque ela é a célula mater responsável pela socialização dos indivíduos. $M$ as como se viu neste texto, a problemática não se reduz ao contexto familiar. 0 indivíduo, inserido numa rede de relações, vive no contexto sociocultural ehistórico. M as a família tem um papel crucial: quando cuidadora, afetiva, amorosa e comunicativa, possui mais chances de promover condições de possibilidades para o desenvolvimento saudável dos filhos. Por isso, os programas de prevenção de uso de drogas precisam prever aplicações práticas de orientação familiar. Papel fundamental pode ser exercido pelos pediatras que acompanham o desenvolvimento e 0 crescimento da criança (Patton, 1995).

A idéia, quase uma ideologia subentendida no slogan "diga não às drogas", é um americanismo que não condiz com a proposta de redução de danos e de promoção da resiliência. Experimentar drogas lícitas ou ilícitas e usá-las socialmente são atitudes que fazem parte de culturas milenares e é um fato na atualidade. Segundo Lewin (1924), pelo menos 1/4 da humanidade usa al gum tipo de droga.

Um programa compreensivo e voltado à promoção da saúde precisa entender essa quase inevitabilidade com a qual convive o ser humano de buscar algum tipo de prazer em substâncias que produzem algum tipo de sensação. E entender também que a prevenção do abuso de drogas é sinônimo de vida saudável, empreendimento tão importante para os jovens que deve incluir a família, a escola, o grupo de pares, a comunidade e a mídia. Tal abordagem requer uma difícil mas factível articulação dos serviços social, educacional e de saúde, numa visão multidisciplinar e como responsabilidade, também, da sociedade. 0 "combate às drogas", termo militarista proveniente da ideologia americana e, na maioria das vezes, único e obsessivo foco da ação, não deveria prevalecer. Promover um crescimento e desenvolvimento saudáveis, maior igualdade social e de oportunidades, atuar contra a pobreza e o racismo, voltar-se para o desenvolvimento do protagonismo juvenil são propostas que convergem para o cumprimento do ECA e a favor da democracia. 


\section{Colaboradores}

M Schenker e M CS M inayo participaram da conceituação, revisão bibliográfica, elaboração e construção do artigo.

\section{Referências bibliográficas}

Arthur M W, Hawkins JD, Pollard JA, Catalano RE \& Baglioni Jr. AJ 2002. M easuring risk and protective factors for substance use, delinquency, and other adolescent problem behaviors. Evaluation Review 26(6):575-601.

Assis SG 1999. Traçando caminhos em uma sociedade violenta. Fiocruz, Rio de Janeiro.

Assis SG, Avanci JQ, Silva CM FP, M alaquias JV, Santo, NC \& Oliveira RVC. 2003. A representação social do adolescente: um passo decisivo na promoção da saúde. Ciência \& Saúde Coletiva 8(3):669-680.

Assis SG \& Constantino P 2001. Filhas do mundo: infração juvenil feminina no Rio de Janeiro. Fiocruz, Rio deJaneiro.

Bandura A 1977. Self-efficacy: toward a unifying theory of behavioral change. Psychological Review 84(2):191215.

Baumrind D 1966. Effects of authoritative parental control on child behavior. Child Development 37:887-907.

Baumrind D 1967. Child care practices anteceding three patterns of preschool behavior. Genetic Psychology M onographs 75:43-88.

Beman DS 1995. Risk factors leading to adolescent substance abuse. Adolescence 30(117):201-208.

Bloom B 1996. The home environment and social learning. San Francisco, Jossey-Bass.

Botvin GJ 1986. Substance abuse prevention research: recent developments and future directions. Journal of School Health 56(9):369-374.

Bronfenbrenner U 1986. Ecology of the family as a context for human development: research perspectives. Developmental Psychology 22(6):723-742.

Brook JS, Brook DW, Gordon AS, Whiteman M \& Cohen P 1990. The psychosocial etiology of adolescent drug use: a family interactional approach. Genetic, Social, and General Psychology M onographs 116(2):111-267.

Brown BB, M ounts N, Lamborn SD \& Steinberg L 1993. Parenting practices and peer group affiliation. Child Development 64:467-482.

Bry BH, M CKeon P \& Pandina RJ 1982. Extent of drug use as a function of number of risk factors. Journal of A bnormal Psychology 91(4):273-279.

Chilcoat HD \& Anthony JC 1996. Impact of parenting monitoring on initiation of drug use through late childhood. Journal of the American Academy of Child and Adolescent Psychiatry 35(1):91-100.

Coie JD, Watt NF, West SG, H awkins JD, Asarnow JR et al. 1993. The science of prevention. A conceptual framework and some directions for a national research program. American Psychologist 48(10):10131022.
De M icheli D \& Formigoni M LOS 2002. Are reasons for the first use of drugs and family circumstances predictors of future use patterns? Addictive Behaviors 27:87-100

Ellickson PL \& M orton SC 1999. Identifying adolescents at risk for hard drug use: racial/ethnic variations. Journal of Adolescent $\mathrm{H}$ ealth 25:382-395.

Emery RS \& Forehand R 1996. Parental divorce and children's well-being: a focus on resilience, pp. 64-99. In LR H aggerty, N Sherrod, N Garmezy \& M Rutter M (eds). Stress, risk and resilience in children and adolescents: process, mechanisms and interventions. Cambridge, Cambridge U niversity Press.

Fergusson DM \& H orwood LJ 1999. Prospective childhood predictors of deviant peer affiliations in adolescence. Journal of Child Psychology and Psychiatry and Allied Disciplines 40(4):581-592.

Friedman HL 1989. The health of adolescents: beliefs and behaviour. Social Science and M edicine 29(3):309315, Section C.

Friedman AS, Terras A \& Kreisher C, 1995. Family and client characteristics as predictors of outpatient treatment outcome for adolescent drug abusers. Journal of Substance A buse 7:345-356.

Garmezy N \& Rutter M 1988. Stress, coping and development in children. M cGraw-Hill, Nova York.

Giddens A 1994. Risco, confiança e reflexividade, pp. 207254. In A Giddens, U Beck \& S Lash (orgs.). M odernidade reflexiva. Editora Unesp, São Paulo.

Hawkins JD, Catalano RF \& M iller JY 1992. Risk and protective factors for alcohol and other drug problems in adolescence and early adulthood: implications for substance abuse prevention. Psychological Bulletin 112(1):64-105.

H eidegger M 1980. H eidegger. Editora Abril, São Paulo. (Coleção Os Pensadores).

H offmann JP \& Cerbone FG 2002. Parental substance use disorder and the risk of adolescent drug abuse: na event history analysis. Drug and Alcohol Dependence 66:255-264

Jessor R 1991. Risk behavior in adolescence: a psychosocial framework for understanding and action. Journal of Adolescent $H$ ealth 12:597-605.

Jessor R, Bos JV, Vanderryn J, Costa FM \& Turbin M S 1995. Protective factors in adolescent problem behavior: moderator effects and developmental change. Developmental Psychology 31(6):923-933.

Johnson GM, Shontz FC \& Locke TP 1984. Relationships between adolescent drug use and parental drug behaviors. Adolescence 19(74):295-299.

Kaminski RA, Stormshak EA, Good III RH \& Goodman 
M R 2002. Prevention of substance abuse with rural head start children and families: results of project STAR. Psychology of Addictive Behaviors 16(4S) :S11S26.

Kandel DB 1990. Parenting styles, drug use, and children's adjustement in families of young adults. Journal of $M$ arriage and the Family 52:183-196.

Kandel DB, Kessler RC \& M argulies RZ 1978. Antecedents of adolescent initiation into stages of drug use: a developmental analysis. Journal of Youth and Adolescence $7(1): 13-40$.

Kodjo CM \& Klein JD 2002. Prevention and risk of adolescent substance abuse. The role of adolescents, families and communities. The Pediatric Clinics of N orth America 49:257-268.

Lewin L 1924. Phantastica: a classic survey on the use amd abuse of mind-altering plants. N ew Books, Portland.

M cCrimmond KR \& Wehrung DA 1986. Taking risk. The Free Press, N ova York.

Minayo et al. 1999. Fala galera: juventude, violência e cidadania no Rio de Janeiro. Editora Garamond, Rio deJaneiro.

M unist M, Santos H, Kotliarenco M , Ojeda E, Infante F \& Grotberg E 1998. M anual de identificación y promoción de la resiliencia en niños y adolescentes. OPS/ OM S/Fundación Kellog, Washington.

Newcomb M D, M addahian E \& Bentler PM 1986. Risk factors for drug use among adolescents: concurrent and longitudinal analyses. American Journal of Public Health 76(5):525-531.

N jaine K 2004. Violência na mídia sob a ótica dos adolescentes. Tese de doutorado defendida na Escola $\mathrm{Na}$ cional de Saúde Pública, Fiocruz, Rio de Janeiro.

O etting ER, D effenbacher JL \& D onnermeyer JF 1998. Primary socialization theory: the role played by personal traits in the etiology of drug use and deviance. Part II. Substance U se \& M isuse 33(6):1337-1366.

Oetting ER \& Donnermeyer JF 1998. Primary socialization theory: the etiology of drug use and deviance. Part I. Substance U se \& M isuse 33(4):995-1026.

Parker JG \& Asher SR 1987. Peer relations and later personal adjustment: are low-accepted children at risk? Psychological Bulletin 102(3):357-389.

Patterson GR \& Fleischman MJ 1979. Maintenance of treatment effects: some considerations concerning family systems and follow-up data. Behavior Therapy 10:168-185.

Patterson GR, Chamberlain P \& Reid JB 1982. A comparative evaluation of a parent training program. Behavior Therapy 13:638-650.

Patton LH 1995. Adolescent substance abuse. Risk factors and protective factors. Pediatric Clinics of $\mathrm{N}$ orth America 42(2): 283-293.

Pettit GS, Bates JE \& Dodge KA 1997. Supportive parenting, ecological context, and children's adjustement: a seven-year longitudinal study. Child Development 68(5):908-923.

Pieper M H \& Pieper J 1999. Smart love: the compassionate alternative to discipline that will make you a better parent and your child a better person. Harvard Common Press, Boston.
Pieper M H \& Pieper J 2002. Addicted to unhappiness: free yourself from moods and behaviors that undermine relationships, work and the life you want. M CGraw-Hill, Nova York.

Rutter M 1987. Psychological resilience and protective mechanisms. American Journal of Orthopsychiatry 57, 316-331.

Schenker M \& M inayo M CS 2003. A implicação da família no uso abusivo de drogas: uma revisão crítica. Ciência \& Saúde Coletiva 8(1):299-306.

Schor EL 1996. Adolescent alcohol use: social determinants and the case for early family-centered prevention. Bulletin of the $\mathrm{N}$ ew York A cademy of M edicine 73(2):335-356.

Shedler J \& Block J 1990. Adolescent drug use and psychological health. American Psychologist 45(5):612630.

Steinberg L, Elmen JD \& M ounts NS 1989. Authoritative parenting, psychosocial maturity, and academic success among adolescents. Child Development 60:14241436.

Steinberg L, Lamborn SD, Dornbusch SM \& Darling N 1992. Impact of parenting practices on adolescent achievement: authoritative parenting, school involvement, and encouragement to succeed. Child Development 63:1266-1281.

Steinberg LD, Fletcher A \& Darling A 1994. Parental monitoring and peer influences on adolescent substance use. Pediatrics 93(6):1060-1064.

Swadi H 1999. Individual risk factors for adolescent substance use. Drug and Alcohol Dependence 55:209-224.

Tarter RE, Sambrano S \& Dunn M G 2002. Predictor variables by developmental stages: a center for substance abuse prevention multisite study. Psychology of Addictive Behaviors 16(4S):S3-S10.

Tec N 1974. Parent-child drug abuse: generational continuity or adolescent deviancy. Adolescence 9:351-364.

Tuttle J, M elnyk BM \& Loveland-Cherry C 2002. Adolescent drug and alcohol use: Strategies for assessment, intervention, and prevention. The N ursing Clinics of N orth America 37:443-460.

Velho G 1998. N obres e anjos: um estudo de tóxicos e hierarquias. Editora FGV, Rio de Janeiro.

Wallace Jr JM 1999. The social ecology of addiction: race, risk, and resilience. Pediatrics 103:1122-1127.

Werner EE 1993. Risk, resilience and recovery. Development and Psychopathology fall, (5):503-515.

Werner EE 1995. Resilience and development. Current Directions in Psychological Science jun (4):81-85.

Werner EE 1996. Vulnerable but invincible: high risk children from birth to adulthood. European Child \& Adolescent Psychiatry 5: 47-51.

Werner M J, J offe A \& Graham AV 1999. Screening, early identification, and office-based intervention with children and youth living in substance-abusing families. Pediatrics 103:1099-1112.

Zweig J M , Phillips BS \& Lindberg LD 2002. Predicting adolescent profiles of risk: looking beyond demographics. Journal of Adolescent H ealth 31:343-353.

Artigo apresentado em 25/05/2004

Aprovado em 23/10/2004

Versão final apresentada em 23/10/2004 